\title{
MEASUREMENTS OF PASSIVE CORRECTION OF MAGNETIZATION HIGHER MULTIPOLES IN ONE METER LONG DIPOLES
}

M. A. Green, R. F. Althaus P. J. Barale,

R. W. Benjegerdes, W. S. Gilbert, M. I. Green,

R. M. Scanlan, and C. E. Taylor

Lawrence Berkeley Laboratory

University of California

Berkeley, CA 94720

\section{$\underline{\text { Abstract }}$}

The use of passive superconductor to correct the magnetization sextupole and decapole in SSC dipoles appears to be promising. This paper presents the results of a series of experiments of passive superconductor correctors in one meter long dipole magnets. Reduction of the magnetization sextupole by a factor of five to ten has been achieved using the passive superconductor correctors. The magnetization decapole was also reduced. The passive superconductor correctors reduced the sextupole temperature sensitivity by an order of magnitude. Flux creep decay was partially compensated for by the correctors.

\section{Background}

The effect of superconductor magnetization on the quality of the field generated within a superconducting dipole was observed as early as $1970 .^{1}$ The effect of superconductor magnetization on field quality has been modeled using complex current doublet theory. ${ }^{2}$ This mathematical model has been applied to both accelerator dipole and quadrupole magnets. There is good agreement between the calculated and measured magnetization multipoles. ${ }^{3}$

The computer model has been used to calculate several methods of passive correction for accelerator dipoles. These methods include: passive superconductor, ${ }^{4,5}$ ferromagnetic material, 4,6 and oriented permanent magnet material. ${ }^{4}$ This paper describes the Lawrence Berkeley Laboratory (LBL) passive superconductor test program. The use of passive superconductors to correct the magnetization sextupole in a dipole magnet is not new. The concept was first described by Brown and Fisk in $1984 .{ }^{7}$ Fermilab reported a test of the concept in $1986 .^{8}$

Passive superconductor within an accelerator dipole has the following potential advantages: 1) passive superconductor correctors are unpowered straight pieces of superconductor mounted within the magnet bore; 2 ) passive superconductor correction corrects magnetization multipoles; 3 ) passive superconductor correction corrects the field over a wide range of inductions; 4) passive superconductor correction will, at least in part, eliminate the slow changes in the magnetization multipoles due to flux creep. 9,10

The concept of passive superconductor correction was tested in two LBL one meter long dipole magnets. The first dipole had a four centimeter bore with iron far enough from the coil so that iron saturation was not an important factor at the full design field of the magnet. The second dipole has a five centimeter bore with close in iron. Saturation of the iron introduces about 12 units of sextupole as well as reduces the transfer function at full design central induction.

This work is supported by the Office of Energy Research, High Energy Physics Division, U. S. Department of Energy, under Contract No. DE-AC03-76SF00098.

Manuscript received September 24, 1990
The passive correctors fabricated at LBL were made from some old $0.808 \mathrm{~mm}$ diameter pieces of superconductor which have 24 micron diameter filaments and a copper to superconductor ratio of 1.36. These pieces of superconductor, which were 26 inches long, were mounted on the surface of a $34.6 \mathrm{~mm}$ (1.362 inch) outside diameter stainless steel bore tube which was inside the dipole. The correctors extended from one end over two thirds of the magnetic length of the dipoles.

\section{Passive Correction of the D-15-C2 Dipole Magnet}

The LBL D-15-C2 dipole is a dipole magnet with NC-9 conductor cross-section (see Fig. 1). The inner coil conductor has 5 micron filaments and a copper-to-superconductor ratio of 1.48. The outer coil conductor has 6 micron diameter filaments and a copperto-superconductor ratio of 1.77 . The corrector for the D-15-C2 dipole is also shown in Fig. 1. The corrector is symmetric about the midplane and the poles. Twelve $0.808 \mathrm{~mm}$ diameter conductors are located about the midplane and ten $0.808 \mathrm{~mm}$ diameter conductors are located at each pole. The amount of superconductor in the corrector represents about 1.6 percent of the superconductor in the D-15-C2 magnet.

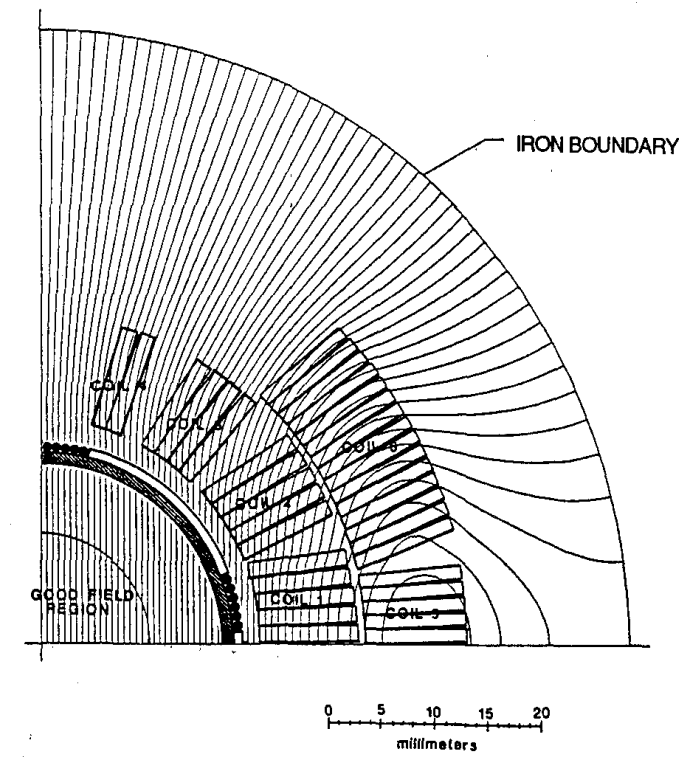

Fig. 1. The D-15-C2 Dipole Coil with the Passive Corrector Installed on the Magnet Bore Tube. 


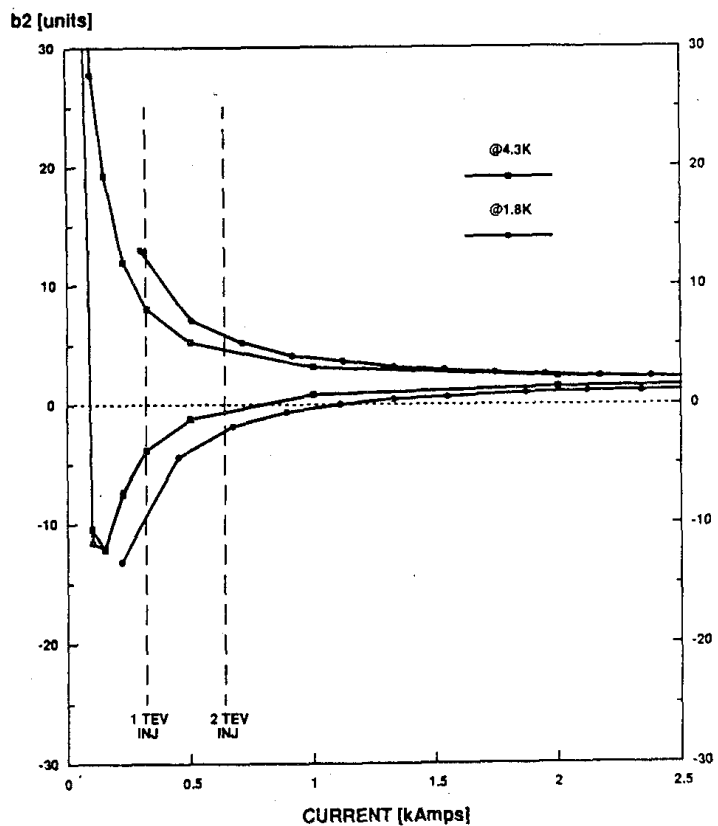

Fig. 2. Magnetic Measurements of the Dipole D-15-C2 Sextupole at $4.3 \mathrm{~K}$ and $1.8 \mathrm{~K}$ without a Passive Corrector.

Figure 2 shows the measured magnetization sextupole of the D15-C2 dipole as a function of current (the dipole transfer function is 10.3 gauss per ampere) without the passive corrector. Figure 3 shows the measured magnetization sextupole for the D-15-C2 dipole as a function of current with the passive corrector installed. The passive superconductor reduced the magnitude of the magnetization sextupole by a factor of five. A comparison of Figs. 2 and 3 shows that the area of the magnetization sextupole hysteresis loop is reduced by the corrector. With the optimal selection of correction superconductor filament diameter, the area of the sextupole hysteresis loop can be reduced to zero over a very wide range of operating currents.

The magnetization sextupole was overcorrected by the passive superconductor corrector. Some of the reasons for the overcorrection of the magnetization sextupole are as follows: 1 ) the actual filament diameter of the corrector was 24 microns instead of the 23 microns assumed when the corrector was designed; 2) the average radius of the corrector mounted on the stainless tube is about $0.1 \mathrm{~mm}$ smaller than the radius used to design the corrector; 3 ) the largest effect is caused by an overestimate of the low field critica current density in the magnet conductor. The low field $\mathrm{J}_{\mathcal{C}}$ of the superconductor in the coil was overestimated by $15 \%$. The errors given above account for over $80 \%$ of the overcorrection observed in the LBL test of dipole D-15-C2.

\section{Passive Correction of the D-16-B Dipole Magne}

The D-16-B1 dipole magnet built by LBL has a 5 centimeter bore with the iron against the outer coil. At low fields, the dipole transfer function is about 13.0 gauss per ampere. The dipole inner coil has 6 micron filaments and a copper to superconductor ratio of 120 . The outer coil has 6 micron filaments and a copper to superconductor ratio of 1.66 .

The D-16-B1 corrector shown in Fig. 4. consists of 28 corrector conductor pieces mounted on the surface of the stainless steel tube. Like the D-15-C2 corrector, the D-16-B1 corrector is symmetric about the midplane and the poles. There are six corrector wires at

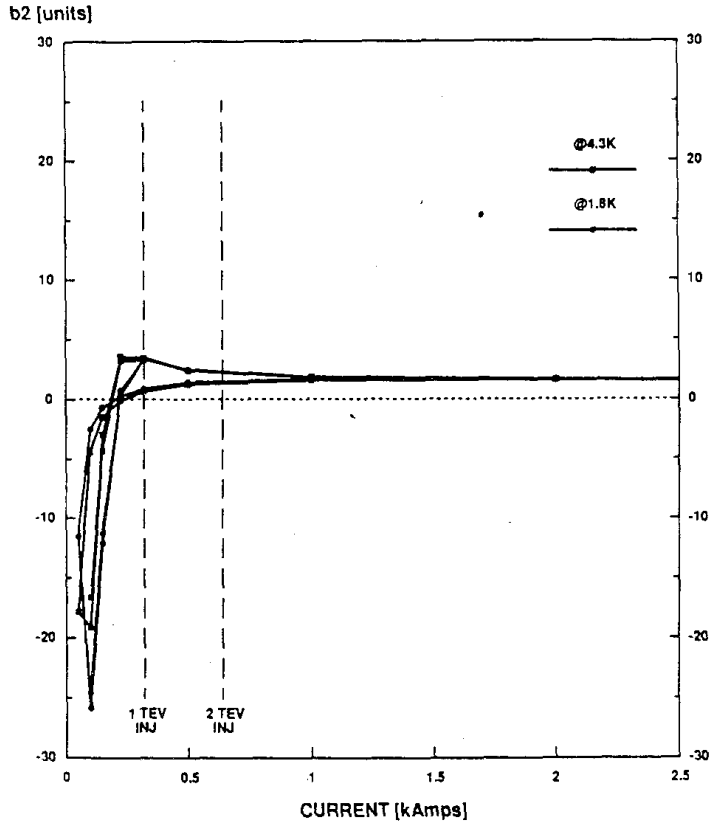

Fig. 3. Magnetic Measurements of the Dipole D-15-C2 Sexrupole at $4.3 \mathrm{~K}$ and $1.8 \mathrm{~K}$ with a Passive Corrector.
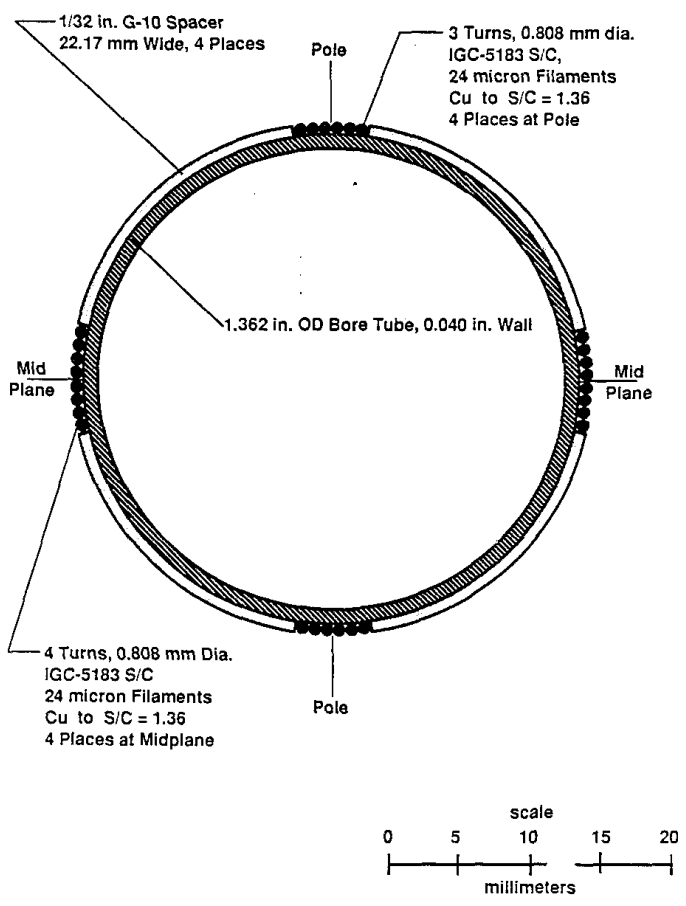

Fig. 4. The Passive Superconductor Corrector for the D-16-B1 Dipole Magnet. 


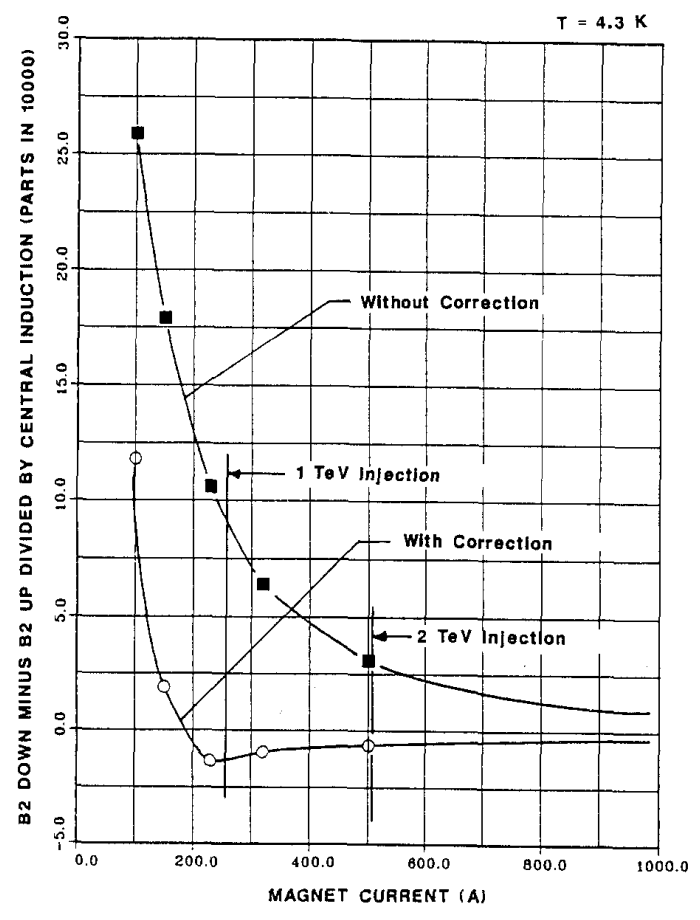

Fig.5. The Width of the Magnetization Sextupole Hystereis Loop with and without Passive Correction as a Function of Current in the D-16-B1 Dipole.

each pole and eight correctors on each side at the midplane. The superconductor in the corrector represents about 0.6 percent of the superconductor in the D-16-B1 magnet.

Figure 5 shows the width of the measured sextupole magnetization curve in the D-16-B1 dipole with and without the passive corrector. Figure 5 demonstrates the extent of correction by the D-16-B1 dipole correctors at the center of the magnet. The curve with correction is negative, which indicates that the corrector overcorrected the magnetization sextupole. The magnitude of the magnetization sextupole was reduced by a factor of seven to eight. (The area of the magnetization sextupole hysteresis loop was reduced by a factor of seven or eight over most of the range of currents.)

\section{Passive Correction of Flux Creep Decay}

Figure 6 compares the decay of the magnetization sextupole in the D-15-C1 dipole without passive correction with the decay of the magnetization sextupole in the $\mathrm{D}-15-\mathrm{C} 2$ dipole with passive superconductor correction. The conductor and the coil designs are the same for the two magnets, so one would expect the two magnets to have the same decay without correction. The sextupole decay measured in the D-15-C1 magnet was 0.75 units per decade; the corrector installed in the D-15-C2 magnet reduced the magnetization decay to +0.15 units per decade. If the flux creep magnetization decay were completely compensated for by the passive corrector, the flux creep decay should have been about -0.15 units per decade. This estimate is based upon the reduction in the magnetization achieved by the passive corrector before the start of the decay. This particular passive corrector is about two thirds as effective in correcting the magnetization decay as it is in correcting the magnetization itself.

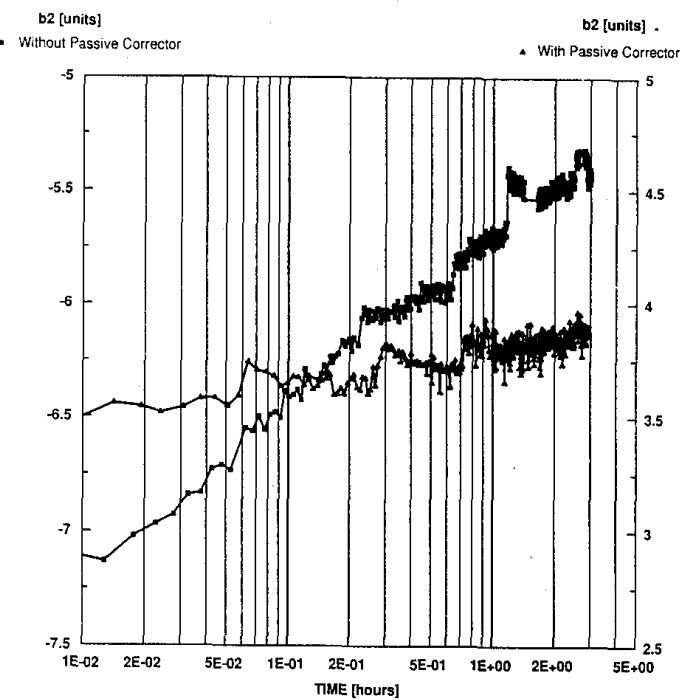

Fig. 6. Magnetization Sextupole Decay in the D-15-C1 Dipole without Passive Correction and Magnetization Sextupole Decay in the D-15-C2 Dipole with Passive Correction.

In the D-16-B1 dipole, the passive superconductor corrector reduced the magnetization sextupole decay about $60 \%$. (The decay rate without the corrector was about +1.0 units per decade. With the corrector, the decay rate was about +0.3 units per decade.) The decay rates at $1.8 \mathrm{~K}$ and $4.3 \mathrm{~K}$ were virtually the same both, with and without the corrector. Temperature appears to have very little effect on magnetization decay as long as it is kept constant during the decay process. The reasons why the passive corrector does not completely correct the magnetization sextupole decay in the same way the magnetization sextupole is corrected is not understood.

Both DESY and LBL have observed that the flux creep decay is different depending on the previous magnetic flux history of the magnet. $11,12,13$ The DESY flux creep decay, made without a passive corrector, with the magnet previously charged to 3000 amperes, is about a factor of 2 smaller than when the magnet has been charged to 6000 amperes. The flux creep decay observed in the D-16-B1 dipole with the passive corrector showed a similar reduction when peak currents were reduced from 6000 amperes to 3000 amperes (see Fig. 7). As discussed above, the magnetization and decays are smaller with the passive corrector but the dependence on peak excitation still exists.

\section{Summary}

The passive corrector experiments on the LBL dipoles demonstrated that correction of magnetization sextupole can be done with pieces of passive superconductor. The magnetization sextupole is corrected by the passive superconductor at a temperature of $1.8 \mathrm{~K}$ as well as $4.3 \mathrm{~K}$ and thus reduces the temperature dependence of the magnetization multipoles. A reduction of the magnetization sextupole was achieved when the field was decreasing as well as when the field was increasing. The decay of the magnetization sextupole was not reduced to the same extent that the magnetization sextupole (at the start of the decay) was reduced.

Passive superconductor correction may be the only correction method which permits the SSC injection energy to be reduced substantially below $2 \mathrm{TeV}$ and still have the quality of magnet field needed to insure that high current proton beams can be injected and stored in the machine. 


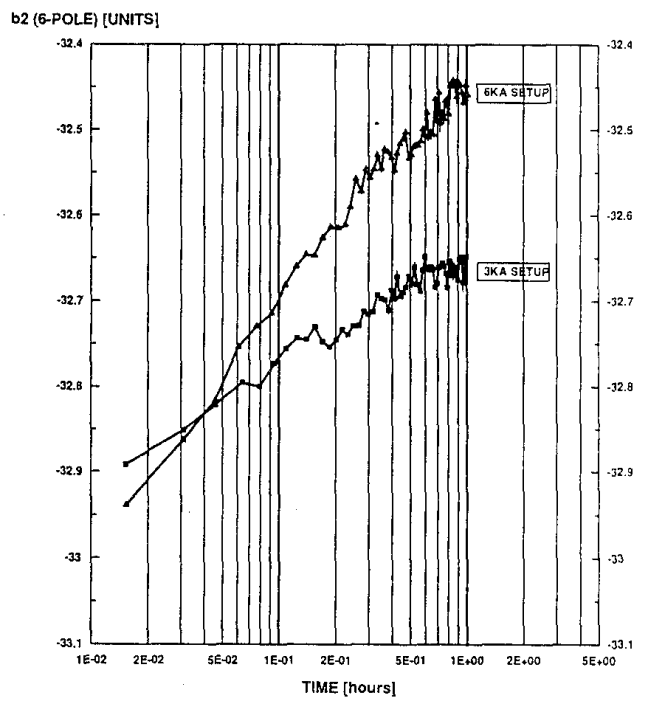

Fig. 7. Magnetization Sextupole Decay in the D-16-B1 Dipole with Passive Correction with 3000 and 6000 Ampere
Peak Dipole Currents.

\section{Acknowledgements}

The authors wish to acknowledge conversations that were had with A. H. Ghosh of Brookhaven National Laboratory concerning the measured magnetization of the superconducting cable used in the test magnets.

\section{$\underline{\text { References }}$}

1. M. A. Green, IEEE Transactions on Nuclear Science NS$18(3), 664,1971$.

2. M. A. Green, "Residual Fields in Superconducting Magnets," Proc. MT-4 Conf., Brookhaven National Laboratory, 339, 1972.

3. M. A. Green, "A Comparison of Calculated and Measured Magnetization Multipoles in the LBL QC-1 One Meter Quadrupole Magnet," Lawrence Berkeley Laboratory LBDD-1627, 1990.

4. M. A. Green, IEEE Transactions on Magnetics MAG-23(2), 506, 1987.

5. M. A. Green, "Passive Superconductor, a Viable Method of Controlling Magnetization Multipoles in the SSC Dipole," IISSC Supercollider 1, Plenum Press, NY, 351, 1989.

6. E. W. Collings, et al., "Design of Multifilamentary Strands for SSC Dipole Magnets," Proc. 1990 ISSC Conf. Miami, FL, 1990.

7. H. C. Brown and H. E. Fisk, the idea of passive superconductor correction presented at the Snowmass SSC Workshop, 1984.

8. H. E. Fisk, et al., report on the Fermilab Passive Superconductor Test, ICFA Workshop, 1986.

9. D. A. Herrup, et al., IEEE Transactions on Magnetics MAG-25(2), 1643, 1989.
10 W. S. Gilbert, et al., Advances in Cryogenic Engineering 36A, 223, Plenum Press, NY, 1990.

11. P. Schmueser of DESY, private communication from slides of his presentation "Persistent Current Effects in the HERA Magnets" at a Topical Workshop on Magnetic Effects of Persistent Currents in Superconductors, Fermilab, 5 March 1990.

12. H. Brück, et al., "Time Dependent Field Distortions from Magnetization Currents in the Superconducting HERA Magnets," to be published in Proc. 13th International Cryogenic Engineering Conf., Beijing, PRC, 25-28 April 1990.

13. W. S. Gilbert, et al., "New Measurements of Magnetic Field Decay in 1 meter SSC Type Dipoles," Proc. 1990 Applied Superconductivity Conf., Snowmass, CO, 1990. 